\title{
A Novel Control Strategy for Three-phase Rectifier
}

\author{
Wei WANG, Xinchun SHI, Jianhui MENG,Chao FU \\ The State Key Laboratory of Alternate Electrical Power System with Renewable Energy Sources \\ North China Electric Power University \\ Bao ding, China \\ E-mail: wangwei_ncepu@163.com
}

\begin{abstract}
Based on the mathematical model analysis of the three-phase voltage source PWM rectifier under abc reference frame and dq reference frame in this paper, a kind of threephase voltage source PWM rectifier feed-forward decoupling control strategy is proposed. The inner current and the voltage loop of double closed loop control strategy is designed. To avoid the output dc voltage fluctuations and improve the system dynamic performance, an adaptive PI control strategy is proposed. It can improve the response of input voltage mutation effectively with the load mutation of the system. The experimental results indicate that this control method has good steady-state performance and fast dynamic response. For the reason of its simpleness, this control method has certain practical value.
\end{abstract}

Keywords- PWM rectifier, adaptive PI controller, dynamic response

\section{INTRODUCTION}

The application of PWM rectifier is becoming more and more widely with the harmonic standard of power equipment more and more strictly. In the control of the PMW rectifier, the most widely used and most excellent performance method is the direct current control in $\mathrm{d}-\mathrm{q}$ synchronous rotating coordinate system at the present study. In the PI control in synchronous rotating reference frame, because of the dc gain of the part of the integral infinite, the converter can realize zero steady-state error. In the structure of the control, double closed loop control which contains the voltage loop and the current inner control is usually adopted. The voltage loop is used to control the output voltage of the rectifier.

When the load is disturbed or changed, fast dynamic response is the most important for high performance of the system. In three-phase PWM rectifier, slow dynamic response may cause voltage too high, and it will be uncontrolled in the process of transient. In generally, it cannot guarantee voltage stability quickly in the dynamic process in synchronous PI control of the rotating coordinate system only considering the steady state performance.

The mathematical model analysis of the three-phase voltage source PWM rectifier under $d-q$ synchronous coordinate system is carried in this paper. Adaptive change of PI parameters to improve the response speed of the dc voltage control method in the process of dynamic is proposed after depth analysis of rapid response of the dc voltage's influence factors. Finally, the experimental results indicate that this method has good steady-state performance and fast dynamic response, and has certain application value.

\section{THE TRANSIENT MATHEMATICAL MODEL OF THE PWM RECTIFIER}

The three-phase voltage source PWM rectifier topology is shown in figure 1 . The physical quantities are defined as follows: $e_{a}, e_{b}$, and $e_{c}$ respectively stand for the three-phase grid voltage. $i_{a}, i_{b}$ and $i_{c}$ respectively stand for the threephase current which flows into the converter. $u_{a}, u_{b}$, and $u_{c}$ respectively stand for AC-side voltage of the converter. $U_{d c}$ stands for DC bus voltage. $L$ is the commutation reactor inductance. $R$ is the equivalent resistance of the switching loss in the converter [1-4].

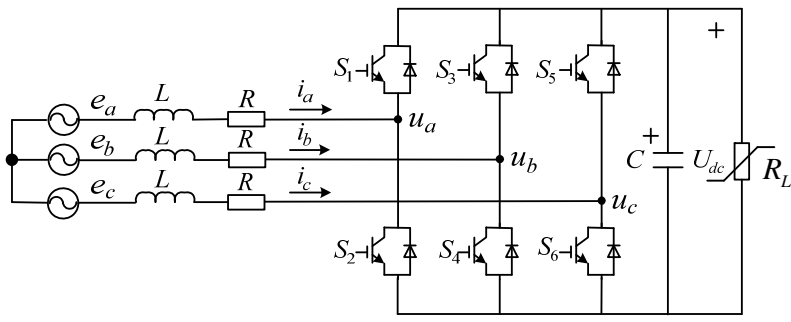

Fig.1. Basic structure of the voltage source converter

A set of differential equations can describe the dynamic model of PWM rectifier.

$$
\left[\begin{array}{l}
\frac{d i_{a}}{d t} \\
\frac{d i_{b}}{d t} \\
\frac{d i_{c}}{d t}
\end{array}\right]=\left[\begin{array}{ccc}
-\frac{R}{L} & 0 & 0 \\
0 & -\frac{R}{L} & 0 \\
0 & 0 & -\frac{R}{L}
\end{array}\right]\left[\begin{array}{l}
i_{a} \\
i_{b} \\
i_{c}
\end{array}\right]+\frac{1}{L}\left[\begin{array}{l}
e_{a}-u_{a} \\
e_{b}-u_{b} \\
e_{c}-u_{c}
\end{array}\right]
$$

According to Park transform, the equation (1) can be represented by following function:

$$
\left[\begin{array}{l}
\frac{d i_{d}}{d t} \\
\frac{d i_{q}}{d t}
\end{array}\right]=\frac{1}{L}\left[\begin{array}{ll}
-R & -\omega L \\
\omega L & -R
\end{array}\right]\left[\begin{array}{l}
i_{d} \\
i_{q}
\end{array}\right]+\frac{1}{L}\left[\begin{array}{l}
e_{d} \\
e_{q}
\end{array}\right]-\frac{1}{L}\left[\begin{array}{l}
u_{d} \\
u_{q}
\end{array}\right]
$$

$e_{d}, e_{q}$ are the d-axis and q-axis component of the grid voltage in (2). $u_{d}, u_{q}$ are the d-axis and q-axis component of AC-side voltage of the converter. $i_{d}, i_{q}$ are the d-axis and q- 
axis component of grid current. $\omega$ is the angular frequency of the grid.

\section{BASED ON ADAPTIVE PI CONTROLLER OF DOUBLE CLOSED LOOP CONTROL STRATEGY}

\section{A. Adaptive PI controller}

The control parameters of the traditional PI controller in the whole control process are constant, and the controller needs to be adjusted when the characteristics of the controlled object changes. But the traditional PI controller does not have this kind of "adaptive" ability. So the PI adaptive quality affects the dynamic performance of the system. The dynamic response can be quickly improved by adaptive setting PI controller parameters according to the running state of the system [5-8].

The adaptive setting integral formula is given in (3).

$$
u_{i}(k)=k_{i}\left\{\sum_{i=0}^{k-1} e(i)+f[e(k)] e(k)\right\} T
$$

The relationship between the coefficient $f$ and the deviation of the current value $|e(k)|$ can be linear or nonlinear.

$$
f[e(k)]=\left\{\begin{array}{cl}
1 & |e(k)| \leq B \\
\frac{A-|e(k)|+B}{A} & B<|e(k)| \leq A+B \\
0 & |e(k)|>A+B
\end{array}\right.
$$

When the current value $|e(k)|$ is greater than the interval of the setting $A+B, f=0$ and the parameters need not to be set. When the current value $|e(k)|$ is less than the interval of the setting $B$, the integral of definite is $u_{i}(k)=k_{i} \sum_{i=0}^{k} e(i) T$. When the current value $|e(k)|$ between $B$ and $A+B$,it is accumulated in parts of current value, the value between 0 $\sim|e(k)|$. It can be changed along with the size of $|e(k)|$.

Seting the values of adaptive setting output as control parameters of the PI controller, using the adaptive setting real-time adjustment, and realizing the PI parameters adaptive tracking the order's change fast. The response speed can be improved. The control output can be represented by following function:

$$
u_{i}(k)=k_{p} e(k)+k_{i}\left\{\sum_{i=0}^{k-1} e(i)+f[e(k)] e(k)\right\} T
$$

The diagram of the adaptive PI controller is shown in figure 2 .

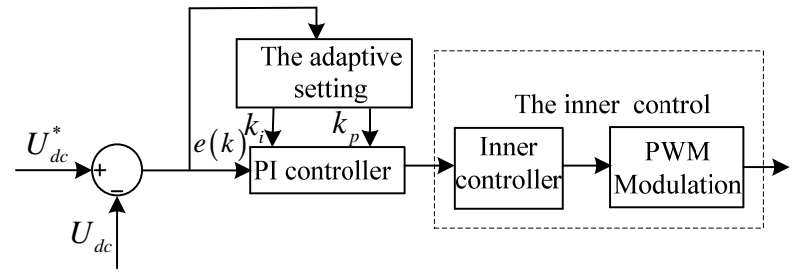

Fig.2. Adaptive PI controller

\section{B. The current inner loop controller}

Combined with (2), the current inner loop mathematical model is:

$$
\left[\begin{array}{l}
e_{d} \\
e_{q}
\end{array}\right]=\left[\begin{array}{lr}
R+L s & \omega L \\
\omega L & R+L s
\end{array}\right]\left[\begin{array}{l}
i_{d} \\
i_{q}
\end{array}\right]+\left[\begin{array}{l}
u_{d} \\
u_{q}
\end{array}\right]
$$

From the d-q model above, the variable of the $d$, q axes are coupling each other, so the controller design is difficult. Therefore, the feed-forward decoupling control strategy can be used to achieve the closed-loop stability control, and the PI regulator can be introducted in the system. The control equation is given (7):

$$
\left\{\begin{array}{l}
u_{d}=-\left(K_{i P}+\frac{K_{i I}}{s}\right)\left(i_{d}^{*}-i_{d}\right)-\omega L i_{q}+e_{d} \\
u_{q}=-\left(K_{i P}+\frac{K_{i I}}{s}\right)\left(i_{q}^{*}-i_{q}\right)+\omega L i_{d}+e_{q}
\end{array}\right.
$$

$K_{i P}$ is the proportion coefficient and $K_{i I}$ is the integral coefficient of current inner loop controller in (7). id*,iq* are the reference value of the current inner loop controller. Combined (6), (7), the transfer function of the current inner loop can be get:

$$
G(S)=\frac{i_{d}}{i_{d}^{*}}=\frac{i_{q}}{i_{q}^{*}}=\frac{K_{i P} s+K_{i I}}{L s^{2}+s\left(K_{i P}+R\right)+K_{i I}}
$$

It can be shown in equation (8) that the current inner can realize decoupling control of active and reactive current by introducing the current state feedback controller of decoupling control strategy. The block diagram of the current inner control is shown in Fig. 3.

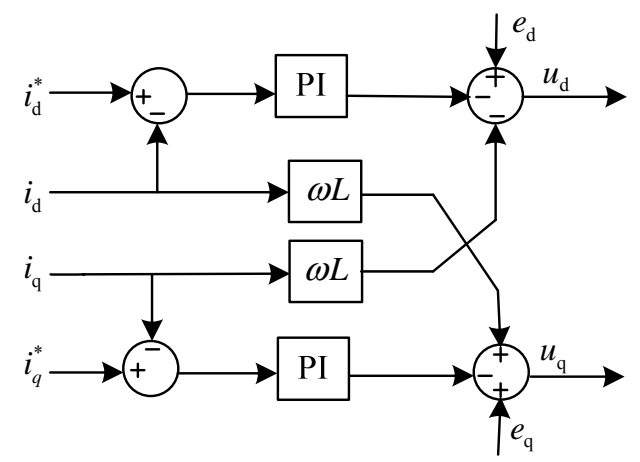

Fig.3. the current inner controller 


\section{EXPERIMENT}

In order to verify the correction of the proposed control strategy of three-phase voltage source PWM rectifier, a physical experimental platform is built. The main parameters of the experimental system are: Grid fundamental frequency is $50 \mathrm{~Hz}$. The transformer is the kind of $220 \mathrm{~V} / 380 \mathrm{~V}$ step-up isolated transformer, the inductance is $1.1 \mathrm{mH}$, and the DC capacitor is $3300 \mu \mathrm{F}$. The IGBT switching frequency is $9 \mathrm{kHz}$, the dead-time is $2 \mu \mathrm{s}$. Master control unit of converter comes from TI company's TMS320F28335, and its CPU clock frequency is $150 \mathrm{MHz}$. This chop is a kind of floating-point chip, compared with previous fixed-point DSP. The chip has characteristics of low cost, high accuracy and low power consumption. In addition, its $\mathrm{A} / \mathrm{D}$ conversion is more accurate and faster and so on.

\section{A. Traditional direct current control}

As is shown in Fig.4, the output voltage of the inverter drops to $70 \%$ of the original at a certain time (from $380 \mathrm{~V}$ to $266 \mathrm{~V})$. It can be seen from Fig.4, the sudden increase of the dc voltage is very high, and the response is very slow.

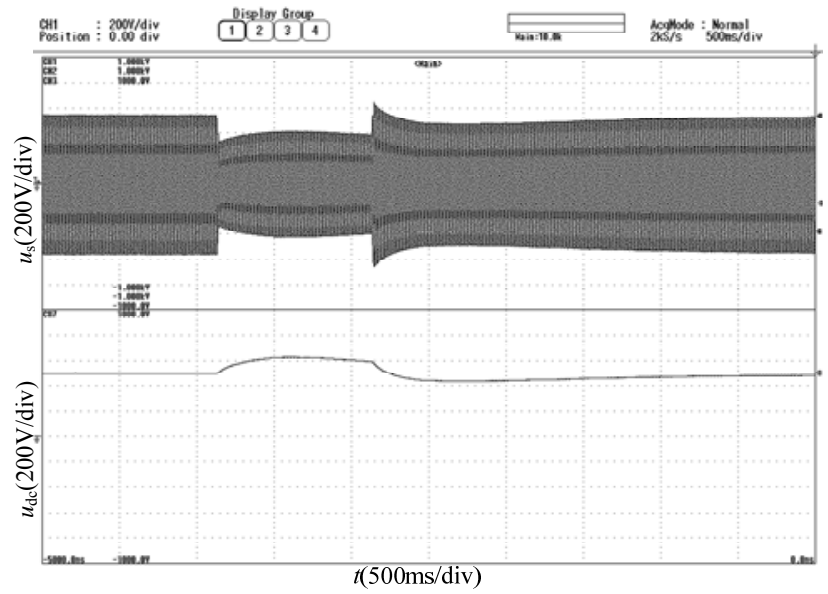

Fig.4.Traditional direct current control

\section{B. Adaptive PI control}

As is shown in Fig.5, the output voltage of the inverter drops to $60 \%$ of the original at a certain time (from $380 \mathrm{~V}$ to $228 \mathrm{~V}$ ), which is shown in Fig.5 (a). It is shown that the output voltage of the inverter drops to $50 \%$ of the original at a certain time (from $380 \mathrm{~V}$ to $190 \mathrm{~V}$ ) in Fig.5 (b). It can be seen from Fig.5, the DC voltage changes little, and the DC voltage rises and keeps stabilization rapidly.

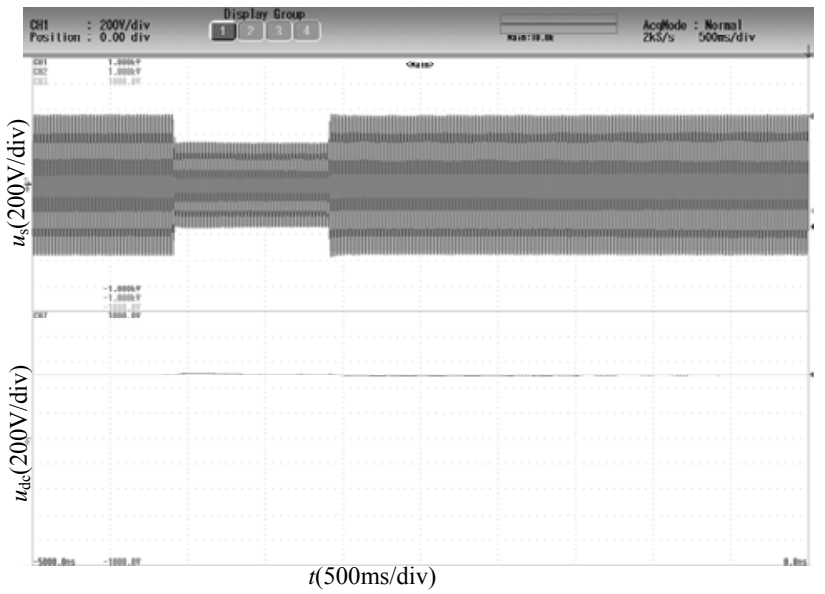

(a)

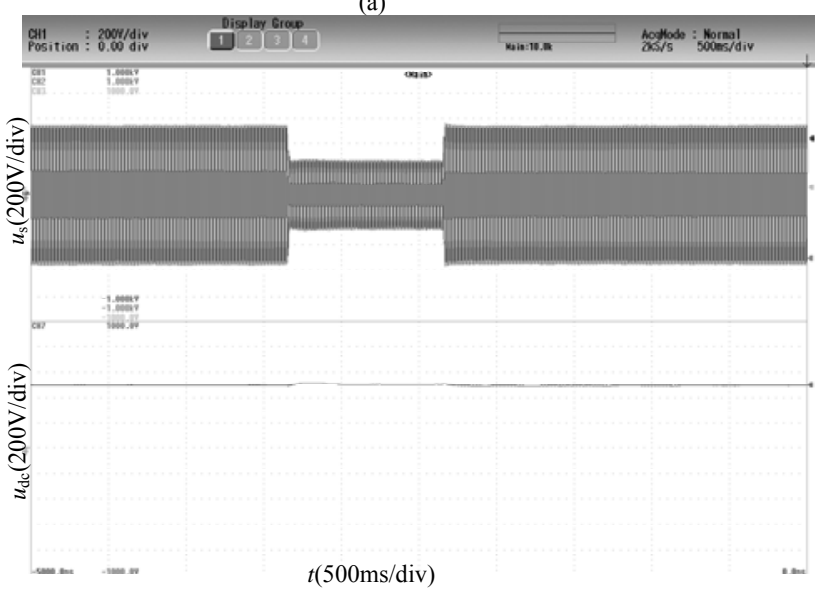

(b)

Fig.5. Adaptive PI control

\section{Comparison}

When lateral load disturbs, the response speed of the dc voltage is slow, and the voltage fluctuation is great under the control in the traditional current. The response speed of $\mathrm{dc}$ voltage is fast, and the voltage fluctuation is very litter. Therefore the system performance is better under the adaptive PI control.

\section{CONCLUSION}

To avoid the shortcomings of the traditional PI controller, the adaptive PI controller is proposed. Adaptive control of parameters setting in PI controller retains the simple structure of PI controller, and the advantages of short time calculation. A comparative analysis is made by experimental research in two aspects of the response speed of the dc voltage stability and the control accuracy between the traditional PI controller and adaptive PI controller. The adaptive PI controller is proved effectively.

\section{REFERENCES}

[1] Vladimir Blasko , Vikram Kaura . A new mathematical model andcontrol of a three-phase ac-dc voltage source converter[J]. IEEE transactions on Power Electronics, 1997, 12(1) : 116-123. 
[2] Zhang Chunjiang, Gu Herong, Wang Baocheng et al. Mathematical model of three-phase PWM rectifier based on a novel phase and amplitude control[J]. Proceedings of the CSEE, 2003, 23(7) : 28-31.

[3] Pan Ching-tsai, Shieh Jenn-jong. New space-vector control strategies for three-phase step up/down AC/DC converter[J]. IEEE transactions on Industrial Electronics, 2000, 47(1) : 25-35.

[4] Mohamad T. Active rectifier using DQ vector control for aircraft power system [C]. IEEE IEMDC, 2007:1306-1310.

[5] YIN Zhi-zhu, YANG Yu-pu, DONG Zu-yi, et al. Doubleclosed-loop fuzzy controlled active power filter[J]. Power System Protection and Control, 2010, 38 (4) : 58-62.
[6] Jean Bosco Mbede, Huang Xinhan, Wang Min. Robust neuro-fuzzy sensor-based motion control among dynamic obstacles for robot manipulators[J]. IEEE Transactions on Fuzzy Systems, 2003, 11 (2) : 249-261.

[7] Salmeron P .Vazquez J R. Practical design of a three-phase active power-line conditioner controlled by artificial neural network[J]. IEEE Transactions on Power Delivery, 2005, 20 (2) : 10371044.

[8] Luo An, Xu Xian-yong, Lu Fang. Feedback-feedforward PI-type iterative learning control strategy for hybrid active power filter with injection circuit[J]. IEEE Transactions on Industrial Electronics, $2010,57(36): 1-11$. 